\title{
THE CONJUGATE HULL OF AN INVERSE SEMIGROUP
}

\author{
by MARIO PETRICH
}

(Received 26 February, 1979)

1. Introduction and summary. There is a large body of literature on inverse semigroups. This literature contains a considerable amount of information concerning congruences on these semigroups, which is not surprising in view of the demonstrated fact that congruences on inverse semigroups play a decisive role in most of the existing structure theorems. In addition, for an inverse semigroup of known structure, finding its congruence lattice, or even certain properties of this lattice, often gives information about these semigroups not apparent in their structure theorems.

Congruences are naturally related to homomorphisms as well as to quotient semigroups. A natural offspring of such considerations is the idea of building inverse semigroups out of the "kernel" and the quotient, that is out of various kinds of extensions. Besides the well-known ideal extensions, which are not specific to inverse semigroups, we have introduced in [6] normal extensions. By definition, a full, self-conjugate inverse subsemigroup $K$ of an inverse semigroup $S$ is a normal subsemigroup of $S$, and $S$ is a normal extension of $K$. In studying these extensions, we have arrived at the normal hull $\Phi(K)$ of $K$ which consists of isomorphisms among subsemigroups of $K$ of the form $e K e$ where $e$ is an idempotent. Normal subsemigroups arise naturally as kernels of homomorphisms on $S$.

If we consider self-conjugate inverse subsemigroups $K$ of $S$, we arrive at the concept of a conjugate extension $S$ of $K$. In this study, the analogue of the translational hull $\Omega(K)$ for ideal extensions and of the normal hull $\Phi(K)$ for normal extensions is the conjugate hull $\Psi(K)$ of $K$. In fact, $\Psi(K)$ consists of all isomorphisms among subsemigroups of $K$ of the form $\lambda K \rho$ where $(\lambda, \rho)$ is an idempotent of the translational hull of $K$. The conjugate hull exhibits several properties analogous to those of $\Omega(K)$ and $\Phi(K)$ for appropriate extensions. The purpose of this paper is to explore some of these properties.

The needed notation and terminology is collected in Section 2. The definition of the conjugate hull and the proof that it is a subsemigroup of the symmetric inverse semigroup takes up Section 3. This is followed in Section 4 by the introduction of conjugate extensions, inner and principal parts of the conjugate hull, and the metacenter, and their relationship is explored. Essential conjugate extensions are briefly considered in Section 5 . The relationship of the conjugate hull and the translational hull is discussed in Section 6 . An embedding of the conjugate hull of a semilattice of groups into its endomorphism semigroup is effected in Section 7. The next, Section 8, contains a construction of the conjugate hull of a Brandt semigroup. The paper is concluded in Section 9 with an example.

2. Notation and terminology. We follow in general the standard notation and terminology of Clifford and Preston [3] and Petrich [5]. We record, however, the following notation most frequently used in the paper.

Glasgow Math. J. 21 (1980) 103-124 
For any semigroup $S, \Omega(S)$ denotes the translational hull of $S, \Pi(S)$ the inner part of $\Omega(S)$, and $\pi: a \rightarrow \pi_{a}=\left(\lambda_{a}, \rho_{a}\right)$ the canonical homomorphism of $S$ into $\Omega(S)$ (or onto $\Pi(S)$ ). For any set $X, \mathscr{I}^{\prime}(X)$ denotes the semigroup of all one-to-one partial transformations on $X$ written on the right, $\mathbf{d} \alpha$ and $\mathbf{r} \alpha$ the domain and range of $\alpha \in \mathscr{I}^{\prime}(X)$, and $\iota_{\mathrm{X}}$ the identity mapping on $X$. For an inverse semigroup $S, E_{S}$ denotes the semilattice of all idempotents of $S$, and $E_{S} \zeta$ the centralizer of $E_{S}$ in $S$.

It is useful to keep in mind that we write left translations on the left and right translations on the right. Hence the notation $\lambda S \rho$ means $\{\lambda s \rho \mid s \in S\}$ where the parentheses in $\lambda s \rho$ are not needed when $S$ is an inverse semigroup. The restriction of a function $\varphi$ to a subset $A$ of its domain is indicated by $\varphi \mid A$.

3. The conjugate hull. Recall that an inverse subsemigroup $K$ of an inverse semigroup $S$ is self-conjugate if $a^{-1} K a \subseteq K$ for all $a \in S$. In this notation, each $a \in S$ induces a function on $K$ defined by $k \rightarrow a^{-1} k a$. This function generally fails to be one-to-one. However, we will take a suitable restriction $\bar{a}$ of this function which is one-to-one. The mapping $a \rightarrow \bar{a}$ associates to each $a \in S$ a one-to-one partial transformation on $K$. We will define below the "conjugate hull" of $K$ which makes it possible to prove that $a \rightarrow \bar{a}$ is actually a homomorphism. In order to do this, we need some preparation.

3.1. Notąion. For any inverse semigroup $S$, let

$\Psi(S)=\left\{\psi \in \mathscr{I}^{\prime}(S) \mid \psi\right.$ is an isomorphism of $\lambda S \rho$ onto $\lambda^{\prime} S \rho^{\prime}$ for some $\left.(\lambda, \rho),\left(\lambda^{\prime}, \rho^{\prime}\right) \in E_{\Omega(S)}\right\}$.

We will have occasion several times to use the following result due to Ault ([1], Proposition 2.3).

3.2. LeMMA. In any inverse semigroup $S$,

$$
\lambda e=e \rho \quad\left(e \in E_{S},(\lambda, \rho) \in E_{\Omega(S)}\right) .
$$

We will use this lemma without specific reference. by

3.3. Lemma. Let $S$ be an inverse semigroup. For any $(\lambda, \rho) \in E_{\Omega(S)}$, define a function $\sigma$

$$
\sigma: a \rightarrow \lambda a \rho \quad(a \in S) .
$$

Then $\sigma \mid E_{\mathrm{S}}$ is a right translation and

$$
a \sigma=\left(a a^{-1}\right) \sigma a\left(a^{-1} a\right) \sigma \quad(a \in S) .
$$

Conversely, if $\sigma$ is a function on $S$ such that $\sigma \mid E_{S}$ is a right translation on $E_{S}$ and (2) holds, then there exists a unique $(\lambda, \rho) \in E_{\Omega(s)}$ such that (1) holds.

Proof. First let $(\lambda, \rho) \in E_{\Omega(S)}$ and define $\sigma$ by (1). Then for any $e, f \in E_{S}$,

$$
(e f) \sigma=\lambda(e f) \rho=(\lambda e)(f \rho)=(e \rho)(f \rho)=e(\lambda f \rho)=e(f \sigma)
$$


so that $\sigma \mid E_{S} \in P\left(E_{S}\right)$. Further, for any $a \in S$,

$$
\begin{aligned}
a \sigma & =\lambda a \rho=\lambda\left[\lambda\left(a a^{-1}\right) a\right] \rho=\lambda\left[\left(a a^{-1}\right) \rho a\right] \rho \\
& =\left[\lambda\left(a a^{-1}\right) \rho\right](a \rho)=\left(a a^{-1}\right) \sigma\left[a\left(a^{-1} a\right) \rho\right] \rho=\left(a^{-1} a\right) \sigma a\left[\lambda\left(a^{-1} a\right)\right] \rho \\
& =\left(a a^{-1}\right) \sigma a\left(a^{-1} a\right) \sigma,
\end{aligned}
$$

as required.

Conversely, let $\sigma$ be a function with the properties enunciated in the statement of the lemma. Define $\lambda$ and $\rho$ by

$$
\lambda a=\left(a a^{-1}\right) \sigma a, \quad a \rho=a\left(a^{-1} a\right) \sigma \quad(a \in S) .
$$

Then

$$
\begin{aligned}
(\lambda a) b & =\left(a a^{-1}\right) \sigma a b=\left[\left(a a^{-1}\right) \sigma(a b)(a b)^{-1}\right](a b) \\
& =\left[(a b)(a b)^{-1}\left(a a^{-1}\right) \sigma\right](a b) \quad\left(\text { since }\left(a a^{-1}\right) \sigma \in E_{S}\right) \\
& =\left[(a b)(a b)^{-1}\left(a a^{-1}\right)\right] \sigma(a b) \\
& =\left[\left(a a^{-1}\right)(a b)(a b)^{-1}\right] \sigma(a b)=\left[(a b)(a b)^{-1}\right] \sigma(a b)=\lambda(a b),
\end{aligned}
$$

symmetrically $a(b \rho)=(a b) \rho$,

$$
\begin{aligned}
a(\lambda b) & =a\left[\left(b b^{-1}\right) \sigma b\right]=a\left[\left(a^{-1} a\right)\left(b b^{-1}\right) \sigma\right] b \\
& =a\left(a^{-1} a\right) \sigma\left(b b^{-1}\right) b=(a \rho) b, \\
\lambda^{2} a & =\lambda(\lambda a)=\lambda\left[\left(a a^{-1}\right) \sigma a\right]=\lambda\left[\left(a a^{-1}\right) \sigma\right] a=\left(a a^{-1}\right) \sigma^{2}\left(a a^{-1}\right) \sigma a \\
& =\left[\left(a a^{-1}\right) \sigma\right]^{2} a=\left(a a^{-1}\right) \sigma a=\lambda a,
\end{aligned}
$$

symmetrically $a \rho^{2}=a \rho$,

$$
\lambda a \rho=\left[\left(a a^{-1}\right) \sigma a\right] \rho=\left(a a^{-1}\right) \sigma(a \rho)=\left(a a^{-1}\right) \sigma a\left(a^{-1} a\right) \sigma=a \sigma,
$$

which establishes all the assertions of the converse except for uniqueness. If $\lambda e \rho=\lambda^{\prime} e \rho^{\prime}$ for some $(\lambda, \rho),\left(\lambda^{\prime}, \rho^{\prime}\right) \in E_{\Omega(s)}$ and all $e \in E$, then

$$
\lambda a=\lambda\left(a a^{-1}\right) a=\left[\lambda\left(a a^{-1}\right) \rho\right] a=\left[\lambda^{\prime}\left(a a^{-1}\right) \rho^{\prime}\right] a=\lambda^{\prime}\left(a a^{-1}\right) a=\lambda^{\prime} a,
$$

so $\lambda=\lambda^{\prime}$, and symmetrically $\rho=\rho^{\prime}$.

3.4. Lemma. Let $\sigma, \sigma^{\prime}, \tau$ be functions on an inverse semigroup $S$ satisfying the conditions in Lemma 3.3. Let $\varphi$ be an isomorphism of $\boldsymbol{S} \sigma$ onto $\boldsymbol{S \sigma}^{\prime}$ and assume that $S \tau \subseteq S \sigma$. Define a function $\tau^{\prime}$ by

$$
\tau^{\prime}: x \rightarrow x \sigma^{\prime} \varphi^{-1} \tau \varphi \quad(x \in S) .
$$

Then $\tau^{\prime}$ satisfies the conditions in Lemma 3.3 and $S \tau \varphi=S \tau^{\prime}$.

Proof. First note that $\tau^{\prime}$ is well-defined since $S \sigma^{\prime}=\mathbf{r} \varphi$ and $S \tau \subseteq S \sigma=d \varphi$. For any 
$e, f \in E_{\mathrm{S}}$, we obtain

$$
\begin{aligned}
(e f) \tau^{\prime} & =(e f) \sigma^{\prime} \varphi^{-1} \tau \varphi=\left[\left(e \sigma^{\prime}\right)\left(f \sigma^{\prime}\right)\right] \varphi^{-1} \tau \varphi \\
& =\left[\left(e \sigma^{\prime} \varphi^{-1}\right)\left(f \sigma^{\prime} \varphi^{-1}\right)\right] \tau \varphi=\left[\left(e \sigma^{\prime} \varphi^{-1}\right)\left(f \sigma^{\prime} \varphi^{-1} \tau\right)\right] \varphi \\
& =\left(e \sigma^{\prime}\right)\left(f \sigma^{\prime} \varphi^{-1} \tau \varphi\right)=e\left(f \sigma^{\prime} \varphi^{-1} \tau \varphi \sigma^{\prime}\right) \\
& =e\left(f \sigma^{\prime} \varphi^{-1} \tau \varphi\right)=e\left(f \tau^{\prime}\right),
\end{aligned}
$$

and hence $\tau^{\prime} \mid E_{S} \in P\left(E_{S}\right)$. Let $\left(\lambda^{\prime}, \rho^{\prime}\right)$ correspond to $\sigma^{\prime}$ and $(\alpha, \beta)$ to $\tau$ as in Lemma 3.3 . Then for any $x \in S$, we get

$$
\begin{aligned}
\left(x x^{-1}\right) \tau^{\prime} x\left(x^{-1} x\right) \tau^{\prime} & =\left[\left(x x^{-1}\right) \sigma^{\prime} \varphi^{-1} \tau \varphi\right] x\left[\left(x^{-1} x\right) \sigma^{\prime} \varphi^{-1} \tau \varphi\right] \\
& =\left[\left(x x^{-1}\right) \sigma^{\prime} \varphi^{-1} \tau \varphi\right] \rho^{\prime} x \lambda^{\prime}\left[\left(x^{-1} x\right) \sigma^{\prime} \varphi^{-1} \tau \varphi\right] \\
& =\left[\left(x x^{-1}\right) \sigma^{\prime} \varphi^{-1} \tau \varphi\right]\left(x \sigma^{\prime}\right)\left[\left(x^{-1} x\right) \sigma^{\prime} \varphi^{-1} \tau \varphi\right] \\
& =\left\{\left[\left(x x^{-1}\right) \sigma^{\prime} \varphi^{-1} \tau\right]\left(x \sigma^{\prime} \varphi^{-1}\right)\left[\left(x^{-1} x\right) \sigma^{\prime} \varphi^{-1} \tau\right]\right\} \varphi \\
& =\left\{\alpha\left[\left(x x^{-1}\right) \sigma^{\prime} \varphi^{-1}\right]\left(x \sigma^{\prime} \varphi^{-1}\right)\left[\left(x^{-1} x\right) \sigma^{\prime} \varphi^{-1}\right] \beta\right\} \varphi \\
& =\left\{\left[\left(x x^{-1}\right) \sigma^{\prime} \varphi^{-1}\right]\left(x \sigma^{\prime} \varphi^{-1}\right)\left[\left(x^{-1} x\right) \sigma^{\prime} \varphi^{-1}\right]\right\} \tau \varphi \\
& =\left\{\left[\left(x x^{-1}\right) \sigma^{\prime}\right]\left(x \sigma^{\prime}\right)\left[\left(x^{-1} x\right) \sigma^{\prime}\right]\right\} \varphi^{-1} \tau \varphi \\
& =x \sigma^{\prime} \varphi^{-1} \tau \varphi=x \tau^{\prime}
\end{aligned}
$$

and $\tau^{\prime}$ satisfies the conditions in Lemma 3.3. Further, for any $x \in S \tau$, we have

$$
x \varphi \tau^{\prime}=(x \varphi) \sigma^{\prime} \varphi^{-1} \tau \varphi=x \varphi \varphi^{-1} \tau \varphi=x \tau \varphi=x \varphi
$$

so that $S \tau \varphi \subseteq S \tau^{\prime}$. Conversely, if $x \in S \tau^{\prime}$, then

$$
x \varphi^{-1} \tau=x \tau^{\prime} \varphi^{-1} \tau=x \sigma^{\prime} \varphi^{-1} \tau \varphi \varphi^{-1} \tau=x \sigma^{\prime} \varphi^{-1} \tau=\left(x \sigma^{\prime} \varphi^{-1} \tau \varphi\right) \varphi^{-1}=x \varphi^{-1}
$$

which shows that $S \tau^{\prime} \subseteq S \tau \varphi$. Consequently $S \tau \varphi=S \tau^{\prime}$.

3.5. Lemma. For any inverse semigroup $S, \Psi(S)$ is a subsemigroup of $\mathscr{I}^{\prime}(S)$.

Proof. Let $\varphi, \psi \in \Psi(S)$ with

$$
\varphi: \lambda S \rho \rightarrow \lambda^{\prime} S \rho^{\prime}, \quad \psi: \alpha S \beta \rightarrow \alpha^{\prime} S \beta^{\prime}
$$

in the obvious notation. Then $\mathbf{d}(\varphi \psi)=(\mathbf{r} \varphi \cap \mathbf{d} \psi) \varphi^{-1}$. Here

$$
\mathbf{r} \varphi \cap \mathbf{d} \psi=\lambda^{\prime} S \rho^{\prime} \cap \alpha S \beta=\lambda^{\prime} \alpha S \beta \rho^{\prime}
$$

since $\left(\lambda^{\prime}, \rho^{\prime}\right),(\alpha, \beta) \in E_{\Omega(S)}$. Hence $\mathbf{d}(\varphi \psi)=\left(\lambda^{\prime} \alpha S \beta \rho^{\prime}\right) \varphi^{-1}$. Now letting

$$
\sigma: a \rightarrow \lambda^{\prime} a \rho^{\prime}, \quad \sigma^{\prime}: a \rightarrow \lambda a \rho, \quad \tau: a \rightarrow \lambda^{\prime} \alpha a \beta \rho^{\prime} \quad(a \in S),
$$

and using $\varphi^{-1}$, we deduce from Lemma 3.4 that $\mathbf{d}(\varphi \psi)=S \tau^{\prime}$ for some $\tau^{\prime}$ satisfying the conditions in Lemma 3.3. Consequently Lemma 3.3 implies that $\mathbf{d}(\varphi \psi)$ is of the form $\xi \mathrm{S} \eta$ for some $(\xi, \eta) \in E_{\Omega(s)}$. Similarly $\mathbf{r}(\varphi \psi)=\left(\lambda^{\prime} \alpha S \beta \rho^{\prime}\right) \psi$ has such a form. 
We thus arrive at the following:

3.6. Definition. For any inverse semigroup $S$, the subsemigroup $\Psi(S)$ of $\mathscr{I}^{\prime}(S)$ consisting of isomorphisms among subsemigroups of $S$ of the form $\lambda S \rho$ with $(\lambda, \rho) \in E_{\mathbf{\Omega}(S)}$ is the conjugate hull of $S$.

In the case when the semigroup $S$ is a semilattice $X, \Psi(S)$ coincides with $U_{X}$ studied by Reilly [8]. If an inverse semigroup $S$ has an identity element, then every bitranslation $(\lambda, \rho)$ is inner, and hence $\Psi(S)$ consists of isomorphisms among subsemigroups of $S$ of the form $e S e$ with $e \in E_{S}$; hence in this case $\Psi(S)$ coincides with $\Phi(S)$ studied in [6]. If in addition, $S=E$ is a semilattice, $\Psi(S)$ coincides with $T_{E}$ introduced by Munn [4].

Since the definition of $\Psi(S)$ is based upon the sets $\lambda S \rho$ with $(\lambda, \rho) \in E_{\Omega(S)}$, these sets deserve at least one more characterization. Recall that a nonempty subset $B$ of a semigroup $S$ is $b i$-ideal of $S$ if $B s B \subseteq B$ for all $s \in S$. Let $X$ be a partially ordered set. For any $x \in X$, the set $[x]=\{y \in X \mid y \leq x\}$ is the principal ideal generated by $x$. A nonempty subset $I$ of $X$ is an (order) ideal of $X$ if $x \in I$ implies $[x] \subseteq I$; if, in addition, $[x] \cap I$ is a principal ideal for all $x \in X$, then $I$ is a $p$-ideal. We consider an inverse semigroup $S$ as partially ordered under $s \leq t$ if $s=e t$ for some $e \in E_{\mathrm{S}}$.

3.7. THEOREM. Let $B$ be a subset of an inverse semigroup $S$. Then $B=\lambda$ So for some $(\lambda, \rho) \in E_{\Omega(S)}$ if and only if $B$ is a bi-ideal and an order p-ideal of $S$ with the property: $b \in B$ implies $b b^{-1}, b^{-1} b \in B$.

Proof. Necessity. Let $(\lambda, \rho) \in E_{\Omega(S)}, B=\lambda S \rho$ and let $x, y, z \in S$. Then

$$
(\lambda x \rho) y(\lambda z \rho)=\lambda[(x \rho) y(\lambda z)] \rho \in B
$$

so that $B$ is a bi-ideal of $S$. If $y \leq x$ and $x \in B$, then $y=e x$ for some $e \in E_{S}$ and thus

$$
y=e x=e(\lambda x \rho)=(e \rho)(x \rho)=(\lambda e)(x \rho)=\lambda(e x) \rho=\lambda y \rho
$$

and hence $y \in B$. Consequently $B$ is an order ideal of $S$. Further,

$$
\lambda x \rho=\lambda\left[\left(x x^{-1}\right) x\left(x^{-1} x\right)\right] \rho=\left[\lambda\left(x x^{-1}\right)\right] x\left[\left(x^{-1} x\right) \rho\right] \leq x
$$

since $\lambda\left(x x^{-1}\right),\left(x^{-1} x\right) \rho \in E_{S}$ so that $[\lambda x \rho] \subseteq B \cap[x]$. Conversely, if $y \in B$ and $y \leq x$, then $y=e x$ for some $e \in E_{S}$, which implies that

$$
y=\lambda y \rho=\lambda(e x) \rho=(\lambda e)(x \rho)=(e \rho)(x \rho)=e(\lambda x \rho) \leq \lambda x \rho
$$

so that $B \cap[x] \subseteq[\lambda x \rho]$. Hence $B$ is a $p$-ideal of $S$. If $x \in B$, then $x=\lambda x=x \rho$ and thus

$$
x x^{-1}=(\lambda x) x^{-1}=\lambda\left(x x^{-1}\right)=\left(x x^{-1}\right) \rho
$$

so that $x x^{-1} \in B$. By symmetry, also $x^{-1} x \in B$.

Sufficiency. Let $B$ have the requisite properties. We define a function $\sigma$ by the requirement

$$
[x \sigma]=B \cap[x] \quad(x \in S)
$$


If $e \in E_{\mathrm{S}}$, then $[e]=E_{\mathrm{S}} e \subseteq E_{\mathrm{S}}$ and thus $e \sigma \in E_{\mathrm{S}}$. For $e, f \in E_{\mathrm{S}}$, we obtain

$$
\begin{gathered}
{[e(f \sigma)]=[e][f \sigma]=[e](B \cap[f])=[e] B \cap[e f],} \\
{[(e f) \sigma]=B \cap[e f] .}
\end{gathered}
$$

Now let $g \in[e] B$ so that $g=t b$ for some $t \leq e$ and $b \in B$. Hence

$$
\mathrm{g}=t b=\left(b b^{-1}\right) t b=b\left(b^{-1} t\right) b \in B S B \subseteq B,
$$

which proves that $[e] B \subseteq B$. Conversely, let $g \in B \cap[e f]$. Then $g=e g \in[e] B$ so that $B \cap[e] \subseteq[e] B$. Relations (1) and (2) now yield $\sigma \mid E_{S} \in P\left(E_{S}\right)$.

Let $y \leq\left(x x^{-1}\right) \sigma x\left(x^{-1} x\right) \sigma$. Using $a E_{S}=E_{S} a$ for any $a \in S$, we obtain

$$
\begin{aligned}
y \in\left(x x^{-1}\right) \sigma x\left(x^{-1} x\right) \sigma E_{S} & =\left[\left(x x^{-1}\right) \sigma E_{S}\right] x\left[\left(x^{-1} x\right) \sigma E_{S}\right] \\
& =\left(x x^{-1} E_{S} \cap B\right) x\left(x^{-1} x E_{S} \cap B\right)
\end{aligned}
$$

which implies $y \in B S B \subseteq B$ and $y \in E_{S} x E_{S}=x E_{S}$. It follows that $y \in[x] \cap B=[x \sigma]$ and hence $y \leq x \sigma$. We have proved that $\left(x x^{-1}\right) \sigma x\left(x^{-1} x\right) \sigma \leq x \sigma$.

In order to prove the opposite inclusion, we first let $y \in\left[x x^{-1}\right] x\left[x^{-1} x\right] \cap B$. Then $y=u x v$ where $u=e x x^{-1}$ and $v=x^{-1} x f$ for some $e, f \in E_{s}$. Hence $y=e x f$ and

$$
\begin{gathered}
y y^{-1}=(\operatorname{exf})(e x f)^{-1}=\operatorname{exf} x^{-1} e \in\left[x x^{-1}\right] \cap B, \\
y^{-1} y=(e x f)^{-1}(e x f)=f x^{-1} e x f \in\left[x^{-1} x\right] \cap B, \\
\left(e x f x^{-1} e\right) x\left(f x^{-1} e x f\right)=e x f\left(x^{-1} x\right)\left(x^{-1} e x\right) f=e x f=y,
\end{gathered}
$$

which shows that $y \in\left(\left[x x^{-1}\right] \cap B\right) x\left(\left[x^{-1} x\right] \cap B\right)$. Using this, we obtain

$$
\begin{aligned}
{[x \sigma] } & =[x] \cap B=\left[\left(x x^{-1}\right) x\left(x^{-1} x\right)\right] \cap B \\
& =\left[x x^{-1}\right] x\left[x^{-1} x\right] \cap B \subseteq\left(\left[x x^{-1}\right] \cap B\right) x\left(\left[x^{-1} x\right] \cap B\right) \\
& =\left[\left(x x^{-1}\right) \sigma\right] x\left[\left(x^{-1} x\right) \sigma\right]=\left[\left(x x^{-1}\right) \sigma x\left(x^{-1} x\right) \sigma\right]
\end{aligned}
$$

which implies $x \sigma \leq\left(x x^{-1}\right) \sigma x\left(x^{-1} x\right) \sigma$.

We have proved that the conditions in Lemma 3.3 are satisfied, which gives that $B=\lambda S \rho$ for some $(\lambda, \rho) \in E_{\mathbf{\Omega}(S)}$.

4. Conjugate extensions. We introduce here a number of new concepts and study their relationship.

4.1. Defintrion. An inverse semigroup $S$ is a conjugate extension of an inverse semigroup $K$ if $K$ is a self-conjugate subsemigroup of $S$.

The relationship between a conjugate extension and the conjugate hull is provided by the following result.

4.2. THEOREM. Let an inverse semigroup $S$ be a conjugate extension of an inverse semigroup $K$. Define a function $\theta=\theta(S: K)$ by

$$
\theta: a \rightarrow \theta^{a} \quad(a \in S)
$$


where $\theta^{a}$ is defined by

$$
\theta^{a}: k \rightarrow a^{-1} k a \quad\left(k \in a K a^{-1}\right) .
$$

Then $\theta$ is a homomorphism of $S$ into $\Psi(S)$.

Proof. Let $a \in S$. Define a function $\sigma$ by

$$
\sigma: k \rightarrow a a^{-1} k a a^{-1} \quad(k \in K) .
$$

It follows immediately that $\sigma$ satisfies the conditions in Lemma 3.3 and $K \sigma=a K a^{-1}$. Now $a^{-1}\left(a K a^{-1}\right) a=a^{-1} K a$ also has the property that $K \sigma^{\prime}=a^{-1} K a$ for some $\sigma^{\prime}$ satisfying the conditions in Lemma 3.3. Let $x, y \in a K a^{-1}$ and assume that $a^{-1} x a=a^{-1} y a$. Then $a a^{-1} x a a^{-1}=a a^{-1} y a a^{-1}$, which yields $x=y$, since $x, y \in a K a^{-1}$. Further,

$$
\left(x \theta^{a}\right)\left(y \theta^{a}\right)=\left(a^{-1} x a\right)\left(a^{-1} y a\right)=a^{-1} x\left(a a^{-1}\right) y a=a^{-1} x y a=(x y) \theta^{a}
$$

since $x=x\left(a a^{-1}\right)$. Consequently $\theta^{a} \in \Psi(K)$.

Now let $a, b \in S$. Then

$$
\begin{aligned}
\mathbf{d}\left(\theta^{a} \theta^{b}\right) & =\left(\mathbf{r} \theta^{a} \cap \mathbf{d} \theta^{b}\right)\left(\theta^{a}\right)^{-1}=a\left(a^{-1} K a \cap b K b^{-1}\right) a^{-1} \\
& =a a^{-1} K a a^{-1} \cap a b K b^{-1} a^{-1}=(a b) K(a b)^{-1}=\mathbf{d} \theta^{a b}
\end{aligned}
$$

and for any $x \in \mathbf{d} \theta^{a b}$, we have

$$
x \theta^{a} \theta^{b}=\left(a^{-1} x a\right) \theta^{b}=b^{-1}\left(a^{-1} x a\right) b=(a b)^{-1} x(a b)=x \theta^{a b} .
$$

Consequently $\theta^{a} \theta^{b}=\theta^{a b}$ and $\theta$ is a homomorphism.

The notation $\theta(S: K)$ introduced in the above theorem will be used throughout the remainder of this paper. Recall that for a congruence $\rho$ on an inverse semigroup $S$, the kernel of $\rho$ is defined as

$$
\operatorname{ker} \rho=\left\{a \in S \mid a \rho e \text { for some } e \in E_{S}\right\} \text {. }
$$

Let the kernel of a homomorphism of $S$ mean the kernel of the induced congruence. For $\theta=\theta(S: S)$, we have introduced in [6] the notation:

$$
\Theta(S)=\left\{\theta^{a} \mid a \in S\right\}, \quad M(S)=\operatorname{ker} \theta .
$$

Call $\Theta(S)$ the inner part of $\Psi(S)$ and $M(S)$ the metacenter of $S$. It follows from ([6], Proposition 1) that

$$
M(S)=\left\{a \in S \mid a a^{-1} x a=a x a^{-1} a \text { for all } x \in S\right\} .
$$

By the same reference, we have that $\theta$ is idempotent separating so that $M(S)=E_{\mathrm{S}}$ if and only if $\theta$ is one-to-one (and thus an isomorphism of $S$ onto $\Theta(S)$ ). After some preparation, we will prove that $\Psi(S)$ is a conjugate extension of $\Theta(S)$. $(\lambda a \rho)^{-1}$.

4.3. Lemma. Let $S$ be an inverse semigroup, $a \in S$ and $(\lambda, \rho) \in E_{\Omega(s)}$. Then $\lambda a^{-1} \rho=$

Proof. Indeed, it was proved in [7] that $(\lambda a)^{-1}=a^{-1} \rho^{-1}$ and $(a \rho)^{-1}=\lambda^{-1} a^{-1}$ whence

$$
(\lambda a \rho)^{-1}=(a \rho)^{-1} \rho^{-1}=\lambda^{-1} a^{-1} \rho^{-1}=\lambda a^{-1} \rho .
$$


4.4. Lemma. Let $S$ be an inverse semigroup, $a \in S$ and $\psi \in \Psi(S)$ with $\mathbf{d} \psi=\lambda S \rho$ $\left((\lambda, \rho) \in E_{\mathbf{\Omega}(S)}\right)$. Then $\psi^{-1} \theta^{a} \psi=\theta^{(\lambda a \rho) \psi}$.

Proof. For $x \in \mathbf{d}\left(\psi^{-1} \theta^{a} \psi\right)$, we obtain

$$
\begin{aligned}
x(\lambda a \rho) \psi[(\lambda a \rho) \psi]^{-1} & =\left[\left(x \psi^{-1}\right)(\lambda a \rho)(\lambda a \rho)^{-1}\right] \psi \\
& =\left[\left(x \psi^{-1} \rho\right)(a \rho)(\lambda a \rho)^{-1}\right] \psi \\
& =\left[\left(x \psi^{-1}\right)(a \rho)(\lambda a \rho)^{-1}\right] \psi \quad\left(\text { since } x \psi^{-1} \in \lambda K \rho\right) \\
& =\left[\left(x \psi^{-1}\right)(a \rho)\left(\lambda a^{-1} \rho\right)\right] \psi \quad(\text { by Lemma 4.3) } \\
& =\left[\left(x \psi^{-1}\right) a\left(\lambda a^{-1} \rho\right)\right] \psi \quad\left(\text { since }(\lambda, \rho) \in E_{\Omega(S)}\right) \\
& =\left\{a\left[a^{-1}\left(x \psi^{-1}\right) a\right] \rho\left(a^{-1} \rho\right)\right\} \psi \quad\left(\text { since } x \psi^{-1} \in a S a^{-1}\right) \\
& =\left[a a^{-1}\left(x \psi^{-1}\right) a\left(a^{-1} \rho\right)\right] \psi \quad\left(\text { since } a^{-1}\left(x \psi^{-1}\right) a \in \lambda S \rho\right) \\
& =\left[\left(x \psi^{-1}\right)\left(a a^{-1}\right) \rho\right] \psi \quad\left(\text { since } x \psi^{-1} \in a S a^{-1}\right) \\
& =\left\{\left[\left(x \psi^{-1}\right) a a^{-1}\right] \rho\right\} \psi \\
& =\left(x \psi^{-1}\right) \rho \psi=x \psi^{-1} \psi=x,
\end{aligned}
$$

and an analogous argument shows that $(\lambda a \rho) \psi[(\lambda a \rho) \psi]^{-1} x=x$. Consequently $x \in \mathbf{d} \theta^{(\lambda a \rho) \psi}$.

Conversely, let $x \in \mathbf{d} \theta^{(\lambda a \rho) \psi}$ so that

$$
x=(\lambda a \rho) \psi[(\lambda a \rho) \psi]^{-1} x(\lambda a \rho) \psi[(\lambda a \rho) \psi]^{-1} .
$$

Since $(\lambda a \rho) \psi \in \mathbf{r} \psi$, it follows that $x \in \mathbf{r} \psi$. Further,

$$
\begin{aligned}
x \psi^{-1} & =(\lambda a \rho)(\lambda a \rho)^{-1}\left(x \psi^{-1}\right)=\left[\lambda\left(a a^{-1}\right)\right](a \rho)(\lambda a \rho)^{-1}\left(x \psi^{-1}\right) \\
& =\left(a a^{-1}\right) \rho(a \rho)(\lambda a \rho)^{-1}\left(x \psi^{-1}\right)=a a^{-1}(\lambda a \rho)(\lambda a \rho)^{-1}\left(x \psi^{-1}\right)=a a^{-1}\left(x \psi^{-1}\right),
\end{aligned}
$$

and symmetrically $x \psi^{-1}=\left(x \psi^{-1}\right) a a^{-1}$, so that $x \psi^{-1} \in a K a^{-1}$. Finally,

$$
\begin{aligned}
\lambda\left[a^{-1}\left(x \psi^{-1}\right) a\right] & =\left(\lambda a^{-1}\right)(\lambda a \rho)(\lambda a \rho)^{-1} a a^{-1}\left(x \psi^{-1}\right) a \\
& =\lambda\left[a^{-1}(\lambda a \rho)(\lambda a \rho)^{-1} a\right] a^{-1}\left(x \psi^{-1}\right) a \\
& =a^{-1}(\lambda a \rho)(\lambda a \rho)^{-1}(a \rho) a^{-1}\left(x \psi^{-1}\right) a \\
& =a^{-1}\left[(\lambda a \rho)\left(\lambda a^{-1} \rho\right)(\lambda a \rho)\right] a^{-1}\left(x \psi^{-1}\right) a \quad \quad(\text { by Lemma 4.3) } \\
& =a^{-1}(\lambda a \rho) a^{-1}\left(x \psi^{-1}\right) a, \quad(\text { by Lemma 4.3) } \\
& =a^{-1}(\lambda a \rho)\left(\lambda a^{-1}\right)\left[\lambda\left(x \psi^{-1}\right)\right] a \\
& =a^{-1}(\lambda a \rho)\left(\lambda a^{-1} \rho\right)\left(x \psi^{-1}\right) a \\
& =a^{-1}\left[(\lambda a \rho)(\lambda a \rho)^{-1}\left(x \psi^{-1}\right)\right] a \quad(\text { by Lemma 4.3) } \\
& =a^{-1}\left(x \psi^{-1}\right) a,
\end{aligned}
$$

and symmetrically $\left[a^{-1}\left(x \psi^{-1}\right) a\right] \rho=a^{-1}\left(x \psi^{-1}\right) a$, so that $x \in \mathbf{d}\left(\psi^{-1} \theta^{a} \psi\right)$. 
THE CONJUGATE HULL OF AN INVERSE SEMIGROUP

We have proved that $\mathbf{d}\left(\psi^{-1} \theta^{a} \psi\right)=\mathbf{d} \theta^{(\lambda a \rho) \psi}$. For any $x$ in this set, we get

$$
\begin{aligned}
x\left(\psi^{-1} \theta^{a} \psi\right) & =\left[a^{-1}\left(x \psi^{-1}\right) a\right] \psi=\left\{\lambda\left\{a^{-1}\left[\lambda\left(x \psi^{-1}\right) \rho\right] a\right\} \rho\right\} \psi \\
& =\left[\left(\lambda a^{-1} \rho\right)\left(x \psi^{-1}\right)(\lambda a \rho)\right] \psi=\left[(\lambda a \rho)^{-1}\left(x \psi^{-1}\right)(\lambda a \rho)\right] \psi \\
& =[(\lambda a \rho) \psi]^{-1} x[(\lambda a \rho) \psi]=x \theta^{(\lambda a \rho) \psi},
\end{aligned}
$$

which completes the proof.

4.5. CoRollary. For any inverse semigroup $S, \Psi(S)$ is a conjugate extension of $\Theta(S)$.

We have introduced in [6] the following concepts. A self-conjugate, full (i.e. $E_{S} \subseteq K$ ) inverse subsemigroup $K$ of an inverse semigroup $S$ is a normal subsemigroup of $S$, and $S$ is a normal extension of $K$. The subsemigroup

$$
\Phi(S)=\left\{\psi \in \Psi(S) \mid \mathbf{d} \psi=e S e, \mathbf{r} \psi=f S f \text { for some } e, f \in E_{S}\right\}
$$

of $\Psi(S)$ is the normal hull of $S$. It seems natural to call $\Phi(S)$ the principal part of $\Psi(S)$. Note that $\Theta(S) \subseteq \Phi(S)$ and that $\psi \in \Psi(S)$ and $\mathbf{d} \psi=e S e$ for $e \in E_{S}$ implies $\psi \in \Phi(S)$.

4.6. Definition. Let an inverse semigroup $S$ be a conjugate extension of an inverse semigroup $K$. The greatest inverse subsemigroup of $S$ containing $K$ as a full subsemigroup is the full closure of $K$ in $S$.

It is easy to verify that if $E_{\mathrm{K}}$ is an ideal of $E_{\mathrm{S}}$, the full closure of $K$ in $S$ is the inverse semigroup

$$
\left\{s \in S \mid s s^{-1}, s^{-1} s \in K\right\} .
$$

Note that this is also the greatest normal extension of $K$ in $S$.

4.7. Proposition. Let $S$ be an inverse semigroup. Then $\Phi(S)$ is the full closure of $\Theta(S)$ in $\Psi(S)$, and is self-conjugate in $\Psi(S)$.

Proof. Let $\varphi \in E_{\Phi(S)}$ with $\mathbf{d} \varphi=\mathbf{r} \varphi=e S e$ where $e \in E_{S}$. Then $\varphi=\iota_{e S e}=\theta^{e}$ which shows that $\Theta(S)$ is full in $\Phi(S)$. Conversely, let $\psi \in \Psi(S)$ be such that $\psi \psi^{-1}=\theta^{e}$ and $\psi^{-1} \psi=\theta^{f}$ for some $e, f \in E_{\mathrm{S}}$. Then

$$
\mathbf{d} \psi=\mathbf{d}\left(\psi \psi^{-1}\right)=\mathbf{d} \theta^{e}=e S e
$$

and thus $\psi \in \Phi(S)$. Hence $\Phi(S)$ is the full closure of $\Theta(S)$ in $\Psi(S)$.

Let $\psi \in \Psi(S), \varphi \in \Phi(S), \mathbf{d} \psi=\lambda S \rho$ where $(\lambda, \rho) \in E_{\mathbf{\Omega}(S)}, \mathbf{d} \varphi=e S e, \mathbf{r} \varphi=f S f$ where $e, f \in E_{\mathrm{S}}$. We know by Theorem 3.7 that $\lambda S \rho$ is a $p$-ideal. Thus $\bar{e}$ and $\bar{f}$ can be defined by the requirements

$$
[\bar{e}]=[e] \cap \lambda S \rho, \quad[\bar{f}]=[f] \cap \lambda S \rho .
$$

Then $\bar{e} \in \lambda S \rho$ and hence also $\bar{e}\left(\bar{f} \varphi^{-1}\right) \in \lambda S \rho$ since $\lambda S \rho$ is an order ideal. We will show that, for $g=\left[\bar{e}\left(\bar{f} \varphi^{-1}\right)\right] \psi$,

$$
\mathbf{d}\left(\psi^{-1} \varphi \psi\right)=[\mathrm{g}]
$$

which suffices to prove the second part of the proposition. 
First let $x \in \mathbf{d}\left(\psi^{-1} \varphi \psi\right)$. Then $x \in \mathbf{r} \psi, \quad x \psi^{-1} \in \mathbf{d} \varphi$ and $x \psi^{-1} \varphi \in \mathbf{d} \psi$. Hence $x \psi^{-1} \epsilon$ $[e] \cap \lambda S \rho=[\bar{e}]$ so that $x \psi^{-1} \leq \bar{e}$. Further, $x \psi^{-1} \varphi \in[f] \cap \lambda S \rho=[\bar{f}]$ which implies $x \psi^{-1} \varphi \leq \bar{f}$ and hence $x \psi^{-1} \leq \bar{f} \varphi^{-1}$. Consequently $x \psi^{-1} \leq \bar{e}\left(\bar{f} \varphi^{-1}\right)$ which yields $x \leq\left[\bar{e}\left(\bar{f} \varphi^{-1}\right)\right] \psi=g$.

Conversely, $g \psi^{-1}=\bar{e}\left(\bar{f} \varphi^{-1}\right) \leq \bar{e} \in \lambda S \rho$ so $g \psi^{-1} \in \mathbf{d} \psi$; also $g \psi^{-1} \leq \bar{f} \varphi^{-1} \in \mathbf{d} \varphi$; finally $x \psi^{-1} \varphi \leq \bar{f}$ which implies that $x \psi^{-1} \varphi \in \mathbf{d} \psi$. It follows that $g \in \mathbf{d}\left(\psi^{-1} \varphi \psi\right)$, which establishes (1).

5. Essential conjugate extensions. We adapt first a universal-algebraic concept to the present situation as follows.

5.1. Definition. Let $S$ be a conjugate extension of an inverse semigroup $K$. Then $S$ is an essential conjugate extension of $K$ if the only congruence on $S$ whose restriction to $K$ is the equality relation on $K$ is the equality relation on $S$. Essential conjugate extensions of $K$ are partially ordered by inclusion.

Note that essential conjugate extensions correspond to essential normal extensions defined in [6] and to dense extensions for ideal extensions, see e.g. [5]. We start with a simple result.

5.2. Proposition. Let $S$ be a conjugate extension of an inverse semigroup $K$. Then $\theta(S: K)$ is one-to-one if and only if the metacenter of $K$ is idempotent and $S$ is an essential conjugate extension of $K$.

Proof. The argument here is identical to that in [6, Proposition 2].

We now come to one of the cardinal properties of the conjugate hull.

5.3. TheORem. Let $S$ be an inverse semigroup with idempotent metacenter. Then $\Psi(S)$ is a maximal essential conjugate extension of $\Theta(S)$. Every inverse subsemigroup of $\Psi(S)$ containing $\Theta(S)$ is an essential conjugate extension of $\Theta(S)$. Every essential conjugate extension of $S$ is isomorphic to a subsemigroup of $\Psi(S)$ containing $\Theta(S)$.

Proof. The argument here goes along the same lines as in the proof of the corresponding statement for normal extensions in [6, Théorème 1], with slight modifications which are left to the reader.

As we just mentioned the corresponding result is valid for normal extensions using $\Phi(S)$ instead of $\Psi(S)$ under the same hypothesis that the metacenter of $S$ be idempotent. The same type of result is valid for dense (ideal) extensions with $\Omega(S)$ taking the place of $\Psi(S)$ under the hypothesis that $S$ be weakly reductive. See [5]. Inspired by this latter example, and the corresponding case in groups and their automorphisms, we propose the following:

5.4. CONJECTURE. If an inverse semigroup $S$ has a maximal essential conjugate extension, then $S$ has idempotent metacenter. 
6. Relationship to the translational hull. Not only is the very definition of the conjugate hull based on the idempotents of the translational hull, but, as we will see in this section, there are many interesting relations between these two hulls. It seems useful to introduce the following concepts.

6.1. Definition. For any inverse semigroup $S$, the elements of

(i) $\Psi(S)$ are local automorphisms of $S$,

(ii) $\Phi(S)$ are principal local automorphisms of $S$,

(iii) $\Theta(S)$ are inner local automorphisms of $S$.

Furthermore, if $K$ is an inverse subsemigroup of $S$, and $\psi \in \Psi(S)$, then $K$ is invariant under $\psi$ if $(\mathbf{d} \psi \cap K) \psi \subseteq K$.

It is easy to verify that an inverse subsemigroup $K$ of $S$ is invariant under all inner local automorphisms if and only if it is self-conjugate in $S$. Letting $S$ be an inverse semigroup with the semilattice of idempotents $E$, we recall the following notation:

$$
\begin{aligned}
E \zeta & =\{s \in S \mid s e=e s \text { for all } e \in E\}, \\
E \zeta \zeta & =\{s \in S \mid s x=x s \text { for all } x \in E \zeta\}, \\
E \omega & =\{s \in S \mid s e=e \text { for some } e \in E\}
\end{aligned}
$$

Recall also that a congruence $\rho$ on $S$ is idempotent separating if $e \rho f$ with $e, f \in E$ implies $e=f$. Observe that $E \zeta$ is the centralizer of idempotents and $E \zeta=\operatorname{ker} \mu$, where $\mu=\mu_{S}$ is the greatest idempotent separating congruence on $S$. Further, $E \zeta \zeta$ is the second centralizer of idempotents of $S$. Finally, $E \omega=\operatorname{ker} \sigma$, where $\sigma=\sigma_{\mathrm{S}}$ is the least group congruence on $S$. Note that

$$
E \subseteq M(S) \subseteq E \zeta \zeta=Z(E \zeta) \subseteq E \zeta
$$

where $Z(T)$ is the center of any semigroup $T$.

6.2. Proposition. For any inverse semigroup $S$ with the semilattice $E$ of idempotents, $E, M(S), E \zeta \zeta, E \zeta$ and $E \omega$ are invariant under all local automorphisms.

Proof. Let $\psi \in \Psi(S)$ with $\psi: \lambda S \rho \rightarrow \alpha S \beta$ where $(\lambda, \rho),(\alpha, \beta) \in E_{\Omega(S)}$. The assertion is trivial for $E$. Let $a \in M(S) \cap \lambda S \rho$ and $s \in S$. Then

$$
\begin{aligned}
(a \psi)(a \psi)^{-1} s(a \psi) & =(a \psi)\left[\left(a^{-1} \psi\right) \beta\right] s[\alpha(a \psi)]=(a \psi)\left(a^{-1} \psi\right)(\alpha s \beta)(a \psi) \\
& =\left[a a^{-1}(\alpha s \beta) \psi^{-1} a\right] \psi=\left[a(\alpha s \beta) \psi^{-1} a^{-1} a\right] \psi \\
& =(a \psi)(\alpha s \beta)\left(a^{-1} \psi\right)(a \psi)=[(a \psi) \beta] s\left[\alpha(a \psi)^{-1}\right](a \psi) \\
& =(a \psi) s(a \psi)^{-1}(a \psi)
\end{aligned}
$$

which shows that $a \psi \in M(S)$. 
Next let $a \in E \zeta \cap \lambda S \rho$. Then for any $e \in E$, we get

$$
\begin{aligned}
(a \psi) e & =[(a \psi) \beta] e=(a \psi)(\alpha e)=\left[a(\alpha e) \psi^{-1}\right] \psi=\left[(\alpha e) \psi^{-1} a\right] \psi \\
& =(\alpha e)(a \psi)=(e \beta)(a \psi)=e[\alpha(a \psi)]=e(\alpha \psi),
\end{aligned}
$$

so that $a \psi \in E \zeta$.

Now let $a \in E \zeta \zeta \cap \lambda S \rho$. Then for any $x \in E \zeta$, it follows from [5, V. 6.4] that $a x=\alpha x \beta=x \beta$ since $E \zeta$ is a semilattice of groups. Using this, the same argument which gave $(a \psi) e=e(a \psi)$ now yields $(a \psi) x=x(a \psi)$. Consequently $a \psi \in E \zeta \zeta$.

Finally, let $a \in E \omega \cap \lambda S \rho$. Then $a e=e$ for some $e \in E$ and thus

$$
(\lambda e) \psi=[\lambda(a e)] \psi=[(\lambda a \rho) e] \psi=[a(\lambda e)] \psi=(a \psi)(\lambda e) \psi
$$

where $(\lambda e) \psi \in E$. Hence $a \psi \in E \omega$.

The next lemma makes it possible to single out a particular subsemigroup of $\Psi(S)$ using the elements of $\Omega(S)$.

6.3. Lemma. Let $S$ be an inverse semigroup. For any $(\lambda, \rho) \in \Omega(S)$, define a function $\delta_{(\lambda, \rho)}$ by

$$
\delta_{(\lambda, \rho)}: x \rightarrow \lambda^{-1} x \rho \quad \text { if } \quad x \in \mathbf{d} \delta_{(\lambda, \rho)}=\lambda S \rho^{-1} .
$$

Then $\delta_{(\lambda, \rho)} \in \Psi(S)$.

Proof. First note that

$$
\mathbf{d} \delta_{(\lambda, \rho)}=\lambda S \rho^{-1}=\lambda \lambda^{-1} S \rho \rho^{-1}
$$

with $\left(\lambda \lambda^{-1}, \rho \rho^{-1}\right) \in E_{\Omega(S)}$; analogously $\mathbf{r} \delta_{(\lambda, \rho)}=\lambda^{-1} \lambda S \rho^{-1} \rho$ with $\left(\lambda^{-1} \lambda, \rho^{-1} \rho\right) \in E_{\Omega(S)}$. For any $x, y \in \mathbf{d} \delta_{(\lambda, \rho)}$, we obtain

$$
\begin{aligned}
\left(x \delta_{(\lambda, \rho)}\right)\left(y \delta_{(\lambda, \rho)}\right) & =\left(\lambda^{-1} x \rho\right)\left(\lambda^{-1} y \rho\right)=\lambda^{-1}\left(x \rho \rho^{-1}\right)(y \rho) \\
& =\lambda^{-1}(x y) \rho=(x y) \delta_{(\lambda, \rho)} .
\end{aligned}
$$

This shows that $\delta_{(\lambda, \rho)}$ is a homomorphism; it is clear that $\delta_{(\lambda, \rho)}$ is one-to-one and maps $\lambda S \rho^{-1}$ onto $\lambda^{-1} S \rho$. Consequently $\delta_{(\lambda, \rho)} \in \Psi(S)$.

6.4. Notation. For any semigroup $S$, let

$$
\Omega^{\prime}(S)=\left\{\delta_{(\lambda, \rho)} \mid(\lambda, \rho) \in \Omega(S)\right\} .
$$

It is easy to verify that $\delta:(\lambda, \rho) \rightarrow \delta_{(\lambda, p)}$ is actually a homomorphism of $\Omega(S)$ onto $\Omega^{\prime}(S)$ so that the latter is actually an inverse subsemigroup of $\Psi(S)$. However, this will follow easily from the next result. Also note that for any $s \in S, \delta_{\pi_{s}}=\theta^{s}$ where $\theta=\theta(S: S)$. Recall that $\pi: S \rightarrow \Omega(S)$ is defined by $\pi: s \rightarrow \pi_{s}=\left(\lambda_{s}, \rho_{s}\right)$ with $\lambda_{s} x=s x, x \rho_{s}=x s$ for all $x \in S$.

6.5. Theorem. Let $K$ be an inverse subsemigroup of an inverse semigroup $S$. Then $K$ is invariant under all $\psi$ in $\Omega^{\prime}(S)$ if and only if $K \pi$ is self-conjugate in $\Omega(S)$. If this is the case, 
then the diagram

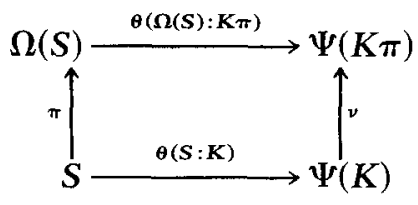

is commutative, where $\nu$ is the isomorphism of $\Psi(K)$ onto $\Psi(K \pi)$ induced by $\pi \mid K$. If $K$ is also full in $S$, then both $\theta(S: K)$ and $\theta(\Omega(S): K \pi)$ are idempotent separating.

Proof. For any $(\lambda, \rho) \in \Omega(S)$, we obtain

$$
\begin{aligned}
\left(\mathbf{d} \delta_{(\lambda, \rho)} \cap K\right) \delta_{(\lambda, \rho)} & =\lambda^{-1}\left(\lambda S \rho^{-1} \cap K\right) \rho=\lambda^{-1} K \rho \subseteq K \\
& \Leftrightarrow(\lambda, \rho)^{-1}(K \pi)(\lambda, \rho) \subseteq K \pi,
\end{aligned}
$$

which proves the first assertion.

Note that for any $\psi \in \Psi(S)$,

$$
\mathbf{d}(\psi \nu)=(\mathbf{d} \psi) \pi, \quad \psi \nu: s \rightarrow s \pi^{-1} \psi \pi \quad \text { if } \quad s \in \mathbf{d}(\psi \nu) .
$$

Assume that $K \pi$ is self-conjugate in $\Omega(S)$. Let $\theta_{1}=\theta(K: S)$ and $\theta_{2}=\theta(\Omega(S): K \pi)$. Then for any $a \in S$, we get

$$
\begin{aligned}
& a \stackrel{\pi}{\longrightarrow} \pi_{a} \stackrel{\theta_{2}}{\longrightarrow} \theta_{2}^{\pi_{a}}, \\
& a \stackrel{\theta_{1}}{\longrightarrow} \theta_{1}^{a} \stackrel{\nu}{\longrightarrow} \theta_{1}^{a} \nu,
\end{aligned}
$$

where

$$
\mathbf{d} \theta_{2}^{\pi_{a}}=\pi_{a}(K \pi) \pi_{a}^{-1}=\left(a K a^{-1}\right) \pi=\mathbf{d}\left(\theta_{1}^{a} \nu\right),
$$

and for any $k \in a K a^{-1}$,

$$
\pi_{k} \theta_{2}^{\pi_{a}}=\pi_{a}^{-1} \pi_{k} \pi_{a}=\pi_{a^{-1} k a}=\pi_{k \theta_{1}{ }^{a}}=k \theta_{1}^{a} \nu,
$$

which establishes the commutativity of the above diagram.

Now suppose that $K$ is full in $S$. First let $e, f \in E_{S}$ be such that $\theta_{1}^{e}=\theta_{1}^{f}$. Then $e K e=f K f$ which implies that $e=e f$ since $e \in E_{\mathrm{S}} \subseteq K$, and analogously $f=f e$, so that $e=f$. Consequently $\theta_{1}=\theta(S: K)$ is idempotent separating.

Next let $(\lambda, \rho),(\alpha, \beta) \in E_{\Omega(s)}$ be such that $\theta_{2}^{(\lambda, \rho)}=\theta_{2}^{(\alpha, \beta)}$. Hence $(\lambda, \rho)(K \pi)(\lambda, \rho)=$ $(\alpha, \beta)(K \pi)(\alpha, \beta)$ so that $\lambda K \rho=\alpha K \beta$. For $e \in E_{S}$, there exists $k \in K$ such that $\lambda e \rho=\alpha k \beta$. It follows that $\lambda e=\alpha \lambda e$, and since this holds for an arbitrary idempotent of $S$, we obtain $\lambda=\alpha \lambda$. By symmetry, $\alpha=\lambda \alpha$ and hence $\lambda=\alpha$, and by symmetry $\rho=\beta$. Hence $(\lambda, \rho)=$ $(\alpha, \beta)$ and $\theta_{2}=\theta(\Omega(S): K \pi)$ is idempotent separating.

6.6. THEOREM. For any inverse semigroup $S$, the function

$$
\delta:(\lambda, \rho) \rightarrow \delta_{(\lambda, \rho)} \quad((\lambda, \rho) \in \Omega(S))
$$


is an idempotent separating homomorphism of $\Omega(S)$ onto $\Omega^{\prime}(S)$ with the kernel $M(\Omega(S))$.

Proof. All assertions except the last one follow from Theorem 6.5 by observing that $\delta=\theta(\Omega(S): \Pi(S)) \nu^{-1}$.

Let $\omega=(\lambda, \rho) \in \Omega(S)$. Then

$$
\begin{aligned}
\omega \in \operatorname{ker} \delta & \Leftrightarrow \delta_{\omega}=\delta_{\varepsilon} \text { for some } \varepsilon=(\alpha, \beta) \in E_{\Omega(S)} \\
& \Leftrightarrow \lambda S \rho^{-1}=\varepsilon S \varepsilon \text { and } \lambda^{-1} a \rho=a \text { if } a \in \varepsilon S \varepsilon \\
& \Leftrightarrow a=\lambda \lambda^{-1} a \rho \rho^{-1} \text { implies } \lambda^{-1} a \rho=a .
\end{aligned}
$$

Assume that the (equivalent) conditions in (1) hold. Since $\delta$ is idempotent separating, we have ker $\delta \subseteq E_{\Omega(S)} \zeta$ which implies that $\omega \omega^{-1}=\omega^{-1} \omega$ since $E_{\Omega(S)} \zeta$ is a semilattice of groups. Hence for any $a \in S$, first $b=\lambda \lambda^{-1} a \rho \rho^{-1}$ has the property $b=\lambda \lambda^{-1} b \rho \rho^{-1}$ so that $\lambda^{-1} b \rho=b$. It follows that

$$
\lambda^{-1}\left(\lambda \lambda^{-1} a \rho \rho^{-1}\right) \rho=\lambda \lambda^{-1} a \rho \rho^{-1}
$$

which implies that $\lambda^{-1} a \rho=\lambda \lambda^{-1} a \rho \rho^{-1}$. Consequently,

$$
\lambda\left(\lambda^{-1} a\right) \rho=\lambda^{2} \lambda^{-1} a \rho \rho^{-1}=\lambda \lambda^{-1} \lambda a \rho^{-1} \rho=\lambda\left(a \rho^{-1}\right) \rho .
$$

In $\Omega(S)$, this can be written as $\omega \omega^{-1} \pi_{a} \omega=\omega \pi_{a} \omega^{-1} \omega$ for all $a \in S$. Now let $\tau \in \Omega(S)$ and $a \in S$. Then

$$
\begin{aligned}
& \left(\omega \omega^{-1} \tau \omega\right) \pi_{a}=\omega \omega^{-1} \tau \omega\left(\omega^{-1} \omega\right) \pi_{a a^{-1}} \pi_{a}=\omega \omega^{-1} \tau \omega \pi_{a a^{-1}} \omega^{-1} \omega \pi_{a} \\
& =\left(\omega \omega^{-1} \pi_{\tau \omega a a^{-1} \omega^{-1}} \omega\right) \pi_{a}=\left(\omega \pi_{\left.\tau \omega a a^{-1} \omega^{-1} \omega^{-1} \omega\right) \pi_{a}}\right. \\
& =\omega \tau\left(\omega \pi_{a a-1} \omega^{-1} \omega\right) \omega^{-1} \pi_{a}=\omega \tau\left(\omega \omega^{-1} \pi_{x a^{-1}} \omega\right) \omega^{-1} \pi_{a} \\
& =\omega \tau \omega \omega^{-1}\left(\pi_{a a^{-1}} \omega \omega^{-1}\right) \pi_{a}=\omega \tau \omega \omega^{-1} \pi_{a}=\left(\omega \tau \omega^{-1} \omega\right) \pi_{a}
\end{aligned}
$$

which implies that $\left(\omega \omega^{-1} \tau \omega\right) a=\left(\omega \tau \omega^{-1} \omega\right) a$, and analogously $a\left(\omega \omega^{-1} \tau \omega\right)=a\left(\omega \tau \omega^{-1} \omega\right)$. This means that $\omega \omega^{-1} \tau \omega=\omega \tau \omega^{-1} \omega$ so that $\omega \in M(\Omega(S))$.

Conversely, let $\omega \in M(\Omega(S))$. Then for any $a \in S$, we have $\lambda\left(\lambda^{-1} a\right) \rho=\lambda\left(a \rho^{-1}\right) \rho$ and $\omega \omega^{-1}=\omega^{-1} \omega$ since $M(\Omega(S)) \subseteq E_{\Omega(S)} \zeta$. For any $a \in S$ such that $a=\lambda \lambda^{-1} a \rho \rho^{-1}$, we get

$$
\lambda^{-1} a \rho=\lambda^{-1}\left[\lambda\left(\lambda^{-1} a\right) \rho\right]=\lambda^{-1}\left[\lambda\left(a \rho^{-1}\right) \rho\right]=\lambda \lambda^{-1} a \rho \rho^{-1}=a,
$$

which in view of (1) yields $\omega \in \operatorname{ker} \delta$.

6.7. Corollary. For any inverse semigroup $S, \theta(\Omega(S): \Omega(S))$ and $\theta(\Omega(S): \Pi(S))$ induce the same congruence on $\Omega(S)$. Moreover, $M(\Omega(S)) \cap \Pi(S)=M(\Pi(S))$.

Proof. The first statement follows from the theorem since

$$
\begin{aligned}
& \operatorname{ker} \theta(\Omega(S): \Omega(S))=M(\Omega(S) \text { (by definition) } \\
& \operatorname{ker} \theta(\Omega(S): \Pi(S))=\operatorname{ker} \delta \quad \text { (since } \nu \text { is an isomorphism), }
\end{aligned}
$$

so the two homomorphisms have the same kernel. Since they are both idempotent separating, the induced congruences must coincide. 
For any $a \in S$, we obtain

$$
\begin{aligned}
\pi_{a} \in M(\Omega(S)) & \Leftrightarrow \pi_{a} \pi_{\alpha}^{-1} \omega \pi_{a}=\pi_{a} \omega \pi_{a^{-1}} \pi_{a} \text { for all } \omega \in \Omega(S) \\
& \Leftrightarrow a\left(a^{-1} \rho\right) a=a\left(\lambda a^{-1}\right) a \text { for all }(\lambda, \rho) \in \Omega(S) \\
& \Leftrightarrow a a^{-1} s a=a s a^{-1} a \text { for all } s \in S \\
& \Leftrightarrow a \in M(S) .
\end{aligned}
$$

If $a \in M(S)$, then $a \in E_{S} \zeta$ so that $a a^{-1}=a^{-1} a$, which for any $(\lambda, \rho) \in \Omega(S)$ gives

$$
a\left(a^{-1} \rho\right) a=a a^{-1}\left[a\left(a^{-1} \rho\right) a\right]=a a^{-1}\left[a\left(\lambda a^{-1}\right) a\right]=a\left(\lambda a^{-1}\right) a .
$$

In view of the above, this establishes the equivalence

$$
\pi_{a} \in M(\Omega(S)) \Leftrightarrow a \in M(S)
$$

which proves the last assertion of the corollary. $E_{\Omega(S)}$

6.8. Corollary. For any inverse semigroup $S, M(S)=E_{S}$ if and only if $M(\Omega(S))=$

Proof. If $M(S)=E_{S}$, then $M(\Pi(S))=E_{\Pi(S)}$ which by the theorem and the above corollary implies that $\delta \mid \Pi(S)$ is one-to-one. Since $\Omega(S)$ is a dense extension of $\Pi(S)$ (see [5, III. 5]), $\delta$ must be one-to-one, so $M(\Omega(S))=E_{\Omega(S)}$. The converse follows directly from the above corollary.

The next result essentially coincides with [8, Theorem 4.4]; we provide here a different setting for it as well as a different proof.

6.9. THEOREM. For any inverse semigroup $S, \xi=\theta\left(\Omega(S): E_{\Pi(S)}\right) \nu^{-1}$ is a homomorphism of $\Omega(S)$ into $\Psi\left(E_{S}\right)$ which induces the greatest idempotent separating congruence on $\Omega(S)$.

Proof. Theorem 6.5 gives that $\xi$ is an idempotent separating homomorphism of $\Omega(S)$ into $\Psi\left(E_{S}\right)$. Let $\omega=(\lambda, \rho) \in E_{\Omega(S)} \zeta$. Then $\omega \varepsilon=\varepsilon \omega$ for all $\varepsilon \in E_{\Omega(S)}$ and, in particular, $\omega \pi_{e}=\pi_{e} \omega$ for all $e \in E_{\mathrm{S}}$. The latter is equivalent to $\lambda e=e \rho$ for all $e \in E_{\mathrm{S}}$. It follows that for any $e \in E_{\mathrm{S}}$,

$$
\lambda^{-1} e=(e \rho)^{-1}=(\lambda e)^{-1}=e \rho^{-1} .
$$

Now $\xi: \omega \rightarrow \xi_{\omega}$ where $\mathbf{d} \xi_{\omega}=\lambda E \rho^{-1}$ and

$$
\xi_{\omega}: e \rightarrow \lambda^{-1} e \rho \quad\left(e \in \lambda E \rho^{-1}\right) .
$$

Using what we just proved, we get $\mathbf{d} \xi_{\omega}=\lambda \lambda^{-1} E$ and $\xi_{\omega}: e \rightarrow \lambda \lambda^{-1} e$ if $e \in \lambda \lambda^{-1} E_{\mathrm{S}}$, which evidently implies that $\xi_{\omega}$ is the identity mapping on its domain. Hence $\xi_{\omega} \in E_{\Psi(s)}$ which yields that $E_{\Omega(S)} \zeta \subseteq \operatorname{ker} \xi$. Since $\xi$ is idempotent separating and $E_{\Omega(S)} \zeta$ is the kernel of the greatest idempotent separating congruence on $\Omega(S)$, we must have $E_{\Omega(S)} \zeta=\operatorname{ker} \xi$.

It follows from Theorem 6.5 that $\pi \xi: S \rightarrow \Psi\left(E_{s}\right)$ is the usual representation of $S$ by its idempotents. 
6.10. Proposrtion. With the notation of Theorem 6.9 , we have $\Pi(S)=\Phi\left(E_{S}\right) \xi^{-1}$.

Proof. For any $a \in S$, we get $\mathbf{d} \xi_{\pi_{\mathrm{a}}}=a E_{S} a^{-1}$ and $\mathbf{r} \xi_{\pi_{a}}=a^{-1} E a$ which shows that $\xi_{\pi_{a}} \in \Phi\left(E_{S}\right)$. Conversely, let $\omega=(\lambda, \rho) \in \Omega(S)$ be such that $\xi_{\omega} \in \Phi\left(E_{S}\right)$. Then $\mathbf{d} \xi_{\omega}=$ $\lambda E_{\mathrm{S}} \rho^{-1}=e E_{\mathrm{S}}$ and $\mathbf{r} \xi_{\omega}=\lambda^{-1} E_{\mathrm{S}} \rho=f E_{\mathrm{S}}$ for some $e, f \in E_{\mathrm{S}}$. In particular, $\lambda^{-1} e \rho=f$ whence

$$
e \rho=e \rho \rho^{-1} \rho=\lambda\left(\lambda^{-1} e \rho\right)=\lambda f=a \text {. }
$$

For any $x \in S$, we obtain

$$
\begin{aligned}
x \rho & =x\left[\left(x^{-1} x\right) \rho \rho^{-1}\right] \rho=x\left[\lambda \lambda^{-1}\left(x^{-1} x\right)\right] \rho \\
& =(x \rho)\left[\lambda^{-1}\left(x^{-1} x\right) \rho\right] f=x\left[\lambda \lambda^{-1}\left(x^{-1} x\right)\right] \rho f \\
& =x\left[\left(x^{-1} x\right) \rho \rho^{-1}\right] \rho f=(x \rho) f=x(\lambda f)=x a
\end{aligned}
$$

and analogously $\lambda x=a x$, which gives $\omega=\pi_{a} \in \Pi(S)$.

6.11. Proposrrion. For any inverse semigroup $S$,

$$
\Phi(S) \cap \Omega^{\prime}(S)=\Theta(S) .
$$

Proof. Let $\omega=(\lambda, \rho) \in \Omega(S)$ be such that $\delta_{\omega} \in \Phi(S)$. Then $\mathbf{d} \delta_{\omega}=\lambda S \rho^{-1}=e S e$ and $\mathbf{r} \delta_{\omega}=\lambda^{-1} S e=f S f$ for some $e, f \in E_{S}$. Letting $a=e \rho$, we obtain

$$
\begin{aligned}
& a a^{-1}=(e \rho)(e \rho)^{-1}=(e \rho)\left(\lambda^{-1} e\right)=\left(e \rho \rho^{-1}\right) e=e \rho \rho^{-1}=e, \\
& a^{-1} a=(e \rho)^{-1}(e \rho)=\left(\lambda^{-1} e\right)(e \rho)=\lambda^{-1} e \rho=f
\end{aligned}
$$

since $e \in \lambda S \rho^{-1}$ implies $e=e \rho \rho^{-1}$; and for $x \in e S e$,

$$
x \delta_{\omega}=\lambda^{-1} x \rho=\lambda^{-1}(e x e) \rho=\left(\lambda^{-1} e\right) x(e \rho)=(e \rho)^{-1} x(e \rho)=a^{-1} e a .
$$

Consequently $\delta_{\omega}=\theta^{a} \in \Theta(S)$. This proves that $\Phi(S) \cap \Omega^{\prime}(S) \subseteq \Theta(S)$; the opposite inclusion is obvious.

7. The conjugate hull of a semilattice of groups. The only result here is that the conjugate hull of a semilattice of groups is embeddable into the endomorphism semigroup of the latter. We have introduced the full closure for inverse semigroups in Definition 4.6; we now extend this concept as follows.

7.1. Definition. Let $A$ be a subsemigroup of a semigroup $S$. If there exists a subsemigroup $B$ of $S$ containing $A$ such that $E_{\mathrm{B}}=E_{\mathrm{A}}$ and $B$ is the greatest subsemigroup of $S$ with this property, then $B$ is the full closure of $A$ in $S$.

It is well-known that a semigroup $S$ which is a semilattice of groups can be given by a system of homomorphisms among these groups, and we use the notation $S=\left[Y ; G_{\alpha}, \varphi_{\alpha, \beta}\right]$. See e.g. [5]. We denote by $\mathscr{E}(S), P(S), \Delta(S)$ the semigroups of all endomorphisms, all right translations, and all inner right translations of any semigroup $S$. For a semilattice $Y$, we write $[\alpha]=\{\beta \in Y \mid \beta \leq \alpha\}$ for any $\alpha \in Y$, and call an ideal $I$ of $Y$ a retract ideal if $I \cap[\alpha]$ is a principal ideal of $Y$ for all $\alpha \in Y$. Denote by $\mathscr{R}_{Y}$ the set of all retract ideals of $Y$ under intersection. For these concepts, consult [5, V.6]. 
7.2. TheORem. Let $S=\left[Y ; G_{\alpha}, \varphi_{\alpha, \beta}\right]$ be a semilattice of groups. Define a function $\chi$ by $\chi: \psi \rightarrow \rho \psi \quad(\psi \in \Psi(S))$

where $\mathbf{d} \psi=\lambda S \rho,(\lambda, \rho) \in E_{\boldsymbol{\Omega}(S)}$. Then $\chi$ is an isomorphism of $\Psi(S)$ into $\mathscr{E}(S)$ such that $\Psi(S) \chi$ is the full closure of $E_{P(S)}, \Phi(S) \chi$ is the full closure of $E_{\Delta(S)}$, and

$$
x: \theta^{a} \rightarrow \rho_{a a^{-1}} \theta^{a} \quad(a \in S) .
$$

Proof. Let $\psi \in \Psi(S)$ and $(\lambda, \rho) \in E_{\mathbf{\Omega}(S)}$ be such that $\mathbf{d} \psi=\lambda S \rho$. It follows from [5, V.6] that for some retract ideal $I$ of $Y$,

$$
x \rho=x \varphi_{\alpha, \bar{\alpha}} \quad \text { if } \quad x \in G_{\alpha}, \quad[\bar{\alpha}]=I \cap[\alpha] .
$$

For any $x \in G_{\alpha}, y \in G_{\beta}$, we obtain

$$
\begin{aligned}
(x \rho)(y \rho) & =\left(x \varphi_{\alpha, \bar{\alpha}}\right)\left(y \varphi_{\beta, \bar{\beta}}\right)=\left(x \varphi_{\alpha, \bar{\alpha} \beta}\right)\left(y \varphi_{\alpha \beta, \overline{\alpha \beta}}\right) \\
& =\left[\left(x \varphi_{\alpha, \alpha \beta}\right)\left(y \varphi_{\beta, \alpha \beta}\right)\right] \varphi_{\alpha \beta, \overline{\alpha \beta}}=(x y) \varphi_{\alpha \beta, \overline{\alpha \beta}} \\
& =(x y) \rho
\end{aligned}
$$

so that $\rho \in \mathscr{E}(S)$. It is now clear that $\rho \psi \in \mathscr{E}(S)$.

Also let $\psi^{\prime} \in \Psi(S)$ with $\mathbf{d} \psi^{\prime}=\lambda^{\prime} S \rho^{\prime},\left(\lambda^{\prime}, \rho^{\prime}\right) \in E_{\Omega(S)}$, and let $I^{\prime}$ be the retract ideal of $Y$ associated with $\rho^{\prime}$ as above. Let $J$ and $J^{\prime}$ be the retract ideals of $Y$ associated with $\mathbf{r} \psi$ and $\mathbf{r} \psi^{\prime}$, respectively, and let

$$
[\hat{\alpha}]=J \cap[\alpha] \quad(\alpha \in Y) .
$$

The functions $\rho, \psi, \rho^{\prime}, \psi^{\prime}$ induce on $Y$ the following functions:

$$
\begin{gathered}
\rho_{1}: \alpha \rightarrow \bar{\alpha}, \quad \rho_{1}^{\prime}: \alpha \rightarrow \hat{\alpha}, \\
\psi_{1}: \alpha \rightarrow \beta \quad \text { if } a \rho=b \quad \text { with } \quad a \in G_{\alpha}, b \in G_{\beta},
\end{gathered}
$$

and $\psi_{1}^{\prime}$ is defined analogously. It is clear that $\rho_{1}, \rho_{1}^{\prime} \in P(Y)$ and $\psi_{1}, \psi_{1}^{\prime} \in \Psi(Y)$, etc. For any $\alpha, \beta \in Y$, we obtain

$$
\begin{array}{rlrl}
\beta \leq \alpha \rho_{1} \psi_{1} \rho_{1}^{\prime} \psi_{1}^{\prime} & \Leftrightarrow \beta \in J^{\prime}, & \beta \psi_{1}^{\prime-1} \leq \alpha \rho_{1} \psi_{1} \rho_{1}^{\prime} & \\
& \Leftrightarrow \beta \in J^{\prime}, \quad \beta \psi_{1}^{\prime-1} \leq \alpha \rho_{1} \psi_{1}, \quad \beta \psi_{1}^{\prime-1} \in I^{\prime} \\
& \Leftrightarrow \beta \in J^{\prime}, \quad \beta \psi_{1}^{\prime-1} \psi^{-1} \leq \alpha \rho_{1}, \quad \beta \psi_{1}^{\prime-1} \in I^{\prime} \\
& \Leftrightarrow \beta \in J^{\prime}, \quad \beta\left(\psi_{1} \psi_{1}^{\prime}\right)^{-1} \leq \alpha, \quad \beta\left(\psi_{1} \psi_{1}^{\prime}\right)^{-1} \in I, \quad \beta \psi_{1}^{\prime-1} \in I^{\prime} \\
& \Leftrightarrow \beta\left(\psi_{1} \psi_{1}^{\prime}\right)^{-1} \leq \alpha \tau_{1}, \quad \beta \in J^{\prime}, \quad \beta \psi_{1}^{\prime-1} \in I^{\prime}, \quad \beta\left(\psi_{1} \psi_{1}^{\prime}\right)^{-1} \in I \\
& \Leftrightarrow \beta \leq \alpha \tau_{1} \psi_{1} \psi_{1}^{\prime}
\end{array}
$$

where $\mathrm{d}\left(\psi \psi^{\prime}\right)=\sigma S \tau,(\sigma, \tau) \in E_{\Omega(S)}$ and $\tau$ induces $\tau_{1}$ as above. It follows that $\rho_{1} \psi_{1} \rho_{1}^{\prime} \psi_{1}^{\prime}=$ $\tau_{1} \psi_{1} \psi_{1}^{\prime}$. Let $K=Y \tau_{1}$.

We return now to $S$. Since $\psi$ is an isomorphism of $\left[I ; G_{\alpha}, \varphi_{\alpha, \beta}\right]$ onto $\left[J ; G_{\alpha}, \varphi_{\alpha, \beta}\right]$, according to [2] there exists a system of isomorphisms

$$
\omega_{\alpha, \alpha \psi_{1}}: G_{\alpha} \rightarrow G_{\alpha \psi_{1}}
$$


such that

and all the diagrams

$$
a \psi=a \omega_{\alpha, \alpha \psi_{1}} \text { if } \quad a \in G_{\alpha}, a \in I
$$

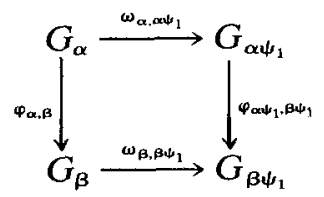

are commutative for $\alpha \geq \beta, \alpha \in I$. The same type of statement is valid for $\psi^{\prime}$ with isomorphisms $\omega_{\alpha, \alpha \psi_{i}^{\prime}}^{\prime}$

Let $\alpha \in Y$. Since $K \subseteq I$, we get $\alpha \tau_{1} \leq \alpha \rho_{1}$. We have seen above that $\alpha \tau_{1} \psi_{1} \psi_{1}^{\prime}=$ $\alpha \rho_{1} \psi_{1} \rho_{1}^{\prime} \psi_{1}^{\prime}$; but $\alpha \tau_{1} \psi_{1} \psi_{1}^{\prime}=\alpha \tau_{1} \psi_{1} \rho_{1}^{\prime} \psi_{1}^{\prime}$ and thus $\alpha \tau_{1} \psi_{1} \rho_{1}^{\prime} \psi_{1}^{\prime}=\alpha \rho_{1} \psi_{1} \rho_{1}^{\prime} \psi_{1}^{\prime}$. This implies that $\alpha \tau_{1} \psi_{1} \rho_{1}^{\prime}=\alpha \rho_{1} \psi_{1} \rho_{1}^{\prime}$. We have the following scheme of mappings.

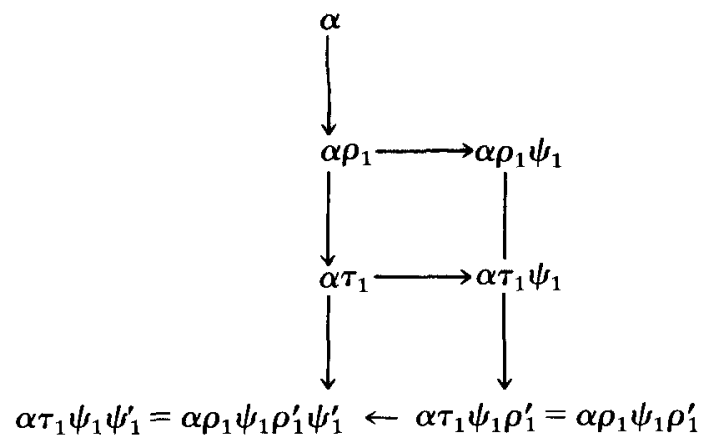

Now substituting $G_{\beta}$ for each element $\beta$ in this diagram, and using the properties of $\omega_{\alpha, \beta}$ and $\omega_{\alpha, \beta}^{\prime}$, we get for any $a \in G_{\alpha}$,

$$
\begin{aligned}
a \rho \psi \rho^{\prime} \psi^{\prime} & =a \varphi_{\alpha, \alpha \rho_{1}} \omega_{\alpha \rho_{1}, \alpha \rho_{1} \psi_{1}} \varphi_{\alpha \rho_{1} \psi_{1}, \alpha \rho_{1} \psi_{1} \rho_{1}^{\prime}} \omega_{\alpha \rho_{1} \psi_{1} \rho_{1}^{\prime}, \alpha \rho_{1} \psi_{1} \rho_{i}^{\prime} \psi_{i}^{\prime}}^{\prime} \\
& =a \varphi_{\alpha, \alpha \rho_{1}} \varphi_{\alpha \rho_{1}, \alpha \tau_{1}} \omega_{\alpha \tau_{1}, \alpha \tau_{1} \psi_{1}} \varphi_{\alpha \tau_{1} \psi_{1}, \alpha \tau_{1} \psi_{1} \rho_{1}} \omega_{\alpha \tau_{1} \psi_{1} \rho_{1}, \alpha \tau_{1} \psi_{1} \psi_{1}^{\prime}}^{\prime} \\
& =a \varphi_{\alpha, \alpha \tau_{1}} \omega_{\alpha \tau_{1}, \alpha \tau_{1} \psi_{1}} \omega_{\alpha \tau_{1} \psi_{1}, \alpha \tau_{1} \psi_{1} \psi_{i}}^{\prime} \\
& =a \tau \psi \psi
\end{aligned}
$$

since $\alpha \tau_{1} \psi_{1} \in I^{\prime}$, so that $\alpha \tau_{1} \psi_{1}=\alpha \tau_{1} \psi_{1} \rho_{1}^{\prime}$. We have proved that $\chi$ is a homomorphism.

With the same notation, assume that $\rho \psi=\rho^{\prime} \psi^{\prime}$. Let $\alpha \in I$. Then $\alpha \rho_{1}^{\prime}=\alpha \rho_{1} \rho_{1}^{\prime}=\left(\alpha \rho_{1}^{\prime}\right) \rho_{1}$ since the idempotents of $\Omega(S)$ commute, and hence $\alpha \rho_{1}^{\prime} \in I$. It follows that

$$
\begin{aligned}
\alpha \rho_{1}^{\prime} & =\alpha \rho_{1}^{\prime} \psi_{1} \psi_{1}^{-1}=\left(\alpha \rho_{1}\right) \rho_{1}^{\prime} \psi_{1} \psi_{1}^{-1}=\left(\alpha \rho_{1}^{\prime}\right)\left(\rho_{1} \psi_{1}\right) \psi_{1}^{-1} \\
& =\left(\alpha \rho_{1}^{\prime}\right)\left(\rho_{1}^{\prime} \psi_{1}^{\prime}\right) \psi_{1}^{-1}=\alpha\left(\rho_{1} \psi_{1}\right) \psi_{1}^{-1}=\alpha \rho_{1}=\alpha
\end{aligned}
$$

and hence $\alpha \in I^{\prime}$. Consequently $I \subseteq I^{\prime}$ and by symmetry also $I^{\prime} \subseteq I$. Now the equation $\rho \psi=\rho^{\prime} \psi^{\prime}$ yields $\psi=\psi^{\prime}$. Hence $\chi$ is one-to-one.

Clearly idempotents of $\Psi(S)_{X}$ coincide with the right translations of $S$. Let $C$ be an inverse subsemigroup of $\mathscr{E}(S)$ with the set of idempotents $E_{\mathbf{P}(S)}$, and let $\xi \in C$. Then $\alpha \alpha^{-1}=\rho$ and $\alpha^{-1} \alpha=\rho^{\prime}$ for some $\rho, \rho^{\prime} \in E_{P(S)}$. Let $\psi=\xi \mid S \rho$. If $x, y \in S \rho$ and $x \psi=y \psi$, then 
$x \xi=y \xi$ so

$$
x=x \rho=x \xi \xi^{-1}=y \xi \xi^{-1}=y \rho=y
$$

and $\psi$ is one-to-one. Further, $\mathbf{d} \psi=S \rho$ and $\mathbf{r} \psi=S \xi=S \rho^{\prime}$ which shows that $\psi \in \Psi(S)$. Finally $\rho \psi=\xi$ which proves that $\xi \in \Psi(S)_{\chi}$. Consequently $C \subseteq \Psi(S)_{\chi}$ which establishes the maximality of $\Psi(S)_{X}$.

Substituting $\rho_{e}$ and $\rho_{f}$ for $\rho$ and $\rho^{\prime}$, respectively, in this argument shows that $\Phi(S) \chi$ is the full closure of $E_{\Delta(S)}$. The last assertion of the theorem is trivial.

Sirjajev [9] made the elements of an inverse semigroup $S$ act on the entire semilattice $E_{S}$ by: $e \rightarrow a e a^{-1}$, and on the basis of this developed a theory of fundamental inverse semigroups. He also established the existence of the full closure of $E_{\Delta(S)}$ in $\mathscr{E}(S)$. The link with the usual treatment of fundamental inverse semigroups through $T_{E}=\Phi\left(E_{S}\right)$ is provided by the preceding theorem applied to $E_{\mathrm{S}}$.

8. The conjugate hull of a Brandt semigroup. We construct below a semigroup $T$ and a homomorphism of $T$ onto the conjugate hull of a Brandt semigroup and characterize the induced congruence.

8.1. Notation. For a nonempty set $I$ and a group $G$, denote by $G^{I}$ the set of all functions mapping the subsets of $I$ (including the empty subset) into $G$ with the multiplication $\varphi . \psi$ where

$$
i(\varphi \cdot \psi)=(i \varphi)(i \psi) \text { for all } i \in \mathbf{d}(\varphi \cdot \psi)=\mathbf{d} \varphi \cap \mathbf{d} \psi
$$

Denote by $\mathscr{A}(G)$ the group of automorphisms of a group $G$ and, for $g \in G$, let $\varepsilon_{\mathrm{g}}$ denote the inner automorphism $x \mapsto \mathrm{g}^{-1} x g(x \in G)$ of $G$.

8.2. TheOREM. Let $S=M^{0}(I, G, I ; \Delta)$ be a Brandt semigroup. Then

$$
T=\left\{(\xi, \omega, \eta) \in \mathscr{I}^{\prime}(I) \times \mathscr{A}(G) \times G^{I} \mid \mathbf{d} \xi=\mathbf{d} \eta\right\} \cup 0
$$

with the multiplication: 0 acts as the zero and

$$
(\xi, \omega, \eta)\left(\xi^{\prime}, \omega^{\prime}, \eta^{\prime}\right)=\left(\xi \xi^{\prime}, \omega \omega^{\prime}, \eta \omega^{\prime} . \xi \eta^{\prime}\right)
$$

is a semigroup. Define a function $\chi$ on $T$ by

$$
\chi:(\xi, \omega, \eta) \rightarrow[\xi, \omega, \eta]
$$

where

$$
\begin{gathered}
\mathrm{d}[\xi, \omega, \eta]=\{(i, g, j) \in S \mid i, j \in \mathbf{d} \xi\} \cup 0 \\
{[\xi, \omega, \eta]:(i, g, j) \rightarrow\left(i \xi,(i \eta)^{-1}(g \omega)(j \eta), j \xi\right), \quad 0 \rightarrow 0 .}
\end{gathered}
$$

Then $\chi$ is a homomorphism of $T$ onto $\Psi(S)$ and

$$
\begin{aligned}
{[\xi, \omega, \eta]=\left[\xi^{\prime}, \omega^{\prime}, \eta^{\prime}\right] \Leftrightarrow \xi=\xi^{\prime} \neq \emptyset, \quad \omega=\omega^{\prime} \varepsilon_{c}, \quad i \eta_{:=}=c^{-1}\left(i \eta^{\prime}\right) } \\
\text { if } \quad i \in \mathbf{d} \xi \quad \text { for some } c \in G, \text { or } \xi=\xi^{\prime}=\emptyset .
\end{aligned}
$$

Proof. The associativity of the multiplication in $T$ follows by a straightforward verification and is omitted. 
Let $(\xi, \omega, \eta) \in T$. Define mappings $\lambda$ and $\rho$ on $S$ by

$$
\lambda(i, g, j)=(i, g, j) \rho=\left\{\begin{array}{l}
(i, \mathrm{~g}, j) \text { if } i, j \in \mathbf{d} \xi \\
0 \text { otherwise }
\end{array}\right.
$$

and $\lambda 0=0 \rho=0$. It follows directly from $[5, \mathrm{~V} .5]$ that $(\lambda, \rho) \in E_{\Omega(S)}$ and clearly $\mathrm{d}[\xi, \omega, \eta]=$ $\lambda S \rho$. Similarly, using $\mathbf{r} \xi$, we can define $\left(\lambda^{\prime}, \rho^{\prime}\right) \in E_{\boldsymbol{\Omega}(S)}$ such that $\mathbf{r}[\xi, \omega, \eta]=\lambda^{\prime} S \boldsymbol{\rho}^{\prime}$. A simple verification shows that $[\xi, \omega, \eta]$ is an isomorphism of $\lambda S \rho$ onto $\lambda^{\prime} S \rho^{\prime}$. Consequently $[\xi, \omega, \eta] \in \Psi(S)$.

Let $(\xi, \omega, \eta),\left(\xi^{\prime}, \omega^{\prime}, \eta^{\prime}\right) \in T$. If $i, j \in \mathbf{d}\left(\xi \xi^{\prime}\right)$, then

$$
\begin{aligned}
(i, g, j)[\xi, \omega, \eta]\left[\xi^{\prime}, \omega^{\prime}, \eta^{\prime}\right] & =\left(i \xi,(i \eta)^{-1}(g \omega)(j \eta), j \xi\right)\left[\xi^{\prime}, \omega^{\prime}, \eta^{\prime}\right] \\
& =\left(i \xi \xi^{\prime},\left(i \xi \eta^{\prime}\right)^{-1}\left(i \eta \omega^{\prime}\right)^{-1}\left(g \omega \omega^{\prime}\right)\left(j \eta \omega^{\prime}\right)\left(j \xi \eta^{\prime}\right), j \xi \xi^{\prime}\right) \\
& =\left(i \xi \xi^{\prime},\left[\left(i \eta \omega^{\prime}\right)\left(i \xi \eta^{\prime}\right)\right]^{-1}\left(g \omega \omega^{\prime}\right)\left[\left(j \eta \omega^{\prime}\right)\left(j \xi \eta^{\prime}\right)\right], j \xi \xi^{\prime}\right) \\
& =(i, g, j)\left[\xi \xi^{\prime}, \omega \omega^{\prime}, \eta \omega^{\prime} . \xi \eta^{\prime}\right],
\end{aligned}
$$

and if $i, j \in \mathbf{d}\left(\xi \xi^{\prime}\right)$ does not hold, then

$$
(i, g, j)[\xi, \omega, \eta]\left[\xi^{\prime}, \omega^{\prime}, \eta^{\prime}\right]=0=(i, g, j)\left[\xi \xi^{\prime}, \omega \omega^{\prime}, \eta \omega^{\prime} . \xi \eta^{\prime}\right] .
$$

Consequently $\chi$ is a homomorphism.

Now let $\psi \in \Psi(S)$ with $\mathbf{d} \psi=\lambda S \rho, \mathbf{r} \psi=\lambda^{\prime} S \rho^{\prime}$ where $(\lambda, \rho),\left(\lambda^{\prime}, \rho^{\prime}\right) \in \Omega(S)$. It follows from [5, V.5] that $\lambda$ and $\rho$ are of the form (3) with $\mathbf{d} \xi$ substituted by some subset $A$ of $I$, and analogously for $\lambda^{\prime}$ and $\rho^{\prime}$. In view of [3, Theorem 3.11], specialized to Brandt semigroups, we deduce that $\psi$ can be represented as in (1) for appropriate $\xi, \omega$ and $\eta$. It follows that $\psi=[\xi, \omega, \eta]$ which proves that $\chi$ maps $T$ onto $\Psi(S)$.

Let $(\xi, \omega, \eta),\left(\xi^{\prime}, \omega^{\prime}, \eta^{\prime}\right) \in T$ where $\xi \neq \emptyset$ and $\xi^{\prime} \neq \emptyset$. Then

$$
\begin{aligned}
{[\xi, \omega, \eta] } & =\left[\xi^{\prime}, \omega^{\prime}, \eta^{\prime}\right] \\
& \Leftrightarrow(i, g, j)[\xi, \omega, \eta]=(i, g, j)\left[\xi^{\prime}, \omega^{\prime}, \eta^{\prime}\right] \quad \text { if } i, j \in \mathbf{d} \xi=\mathbf{d} \xi^{\prime} \\
& \Leftrightarrow \xi=\xi^{\prime} \text { and }(i \eta)^{-1}(g \omega)(j \eta)=\left(i \eta^{\prime}\right)^{-1}\left(g \omega^{\prime}\right)\left(j \eta^{\prime}\right) \quad \text { if } i, j \in \mathbf{d} \xi .
\end{aligned}
$$

Let this be the case. Then letting $g$ be the identity of $G$, we get $(i \eta)^{-1}(j \eta)=\left(i \eta^{\prime}\right)^{-1}\left(j \eta^{\prime}\right)$ which implies that $\left(i \eta^{\prime}\right)(i \eta)^{-1}=\left(j \eta^{\prime}\right)(j \eta)^{-1}$. In the last equation, the left hand side depends only upon $i$ and the right hand side only upon $j$, so both are equal to a constant $c$ in $G$. It follows that

$$
g \omega=(i \eta)\left(i \eta^{\prime}\right)^{-1}\left(g \omega^{\prime}\right)\left(j \eta^{\prime}\right)(j \eta)^{-1}=c^{-1}\left(g \omega^{\prime}\right) c=g \omega^{\prime} \varepsilon_{\mathbf{c}}
$$

and hence $\omega=\omega^{\prime} \varepsilon_{c}$. Furthermore, $\left(i \eta^{\prime}\right)(i \eta)^{-1}=c$ implies $i \eta=c^{-1}\left(i \eta^{\prime}\right)$. We have proved the direct implication in (2); the opposite implication can be verified without difficulty.

9. It is instructive to compute the complete inverse images of $\Theta(S), \Phi(S)$ and $\Omega^{\prime}(S)$ under $X$ in the above theorem. For this we need some notation. For any set $X$, let $|X|$ denote its cardinality. If $\xi \in \mathscr{I}^{\prime}(I)$ has domain $\{i\}$ and range $\{j\}$, write $\xi=\xi_{i j}$. Denote by 
$\Phi(G)$ the group of inner automorphisms of $G\left(\varepsilon_{g}: x \rightarrow g^{-1} x g\right.$ for all $\left.x \in G\right)$. For any subset $A$ of $I$, denote by $\iota$ the function which maps $A$ onto the identity of $G$.

Let $(i, g, j) \in S$. Then a simple argument shows that

$$
\begin{gathered}
\mathbf{d} \theta^{(i, g, j)}=\{(i, h, i) \mid h \in G\} \cup 0, \\
\mathbf{r} \theta^{(i, g, j)}=\{(j, h, j) \mid h \in G\} \cup 0, \\
\theta^{(i, g, j)}:(i, h, i) \rightarrow\left(j, h \varepsilon_{\mathrm{g}}, j\right) \quad(h \in G),
\end{gathered}
$$

which imply that

$$
\begin{aligned}
{\left[\xi_{i i j}, \varepsilon_{\mathrm{g}}, \iota\right] } & =\theta^{(i, \mathbf{g}, j)} \\
\Theta(S) \chi^{-1} & =\{(\xi, \omega, \eta) \in T|| \mathbf{d} \xi \mid \leq 1, \omega \in \mathscr{I}(G)\} \\
& \cong M^{0}(I, \Phi(G), I ; \Delta\} .
\end{aligned}
$$

It now follows without difficulty that

$$
\Phi(S) \chi^{-1}=\{(\xi, \omega, \eta) \in T|| \mathbf{d} \xi \mid \leq 1\} \cong \mathscr{M}^{0}(I, \mathscr{A}(G), I ; \Delta) .
$$

Now let $(\lambda, \rho) \in \Omega(S)$. According to [5, V.3 and V.5], there exists $\beta \in \mathscr{I}^{\prime}(I)$ and a function $\psi: \mathbf{d} \beta \rightarrow G$ such that

$$
\begin{aligned}
& \lambda(i, g, j)=\left\{\begin{array}{l}
\left(i \beta^{-1},\left(i \beta^{-1} \psi\right) g, j\right) \quad \text { if } i \in \mathbf{r} \beta, \\
0 \text { otherwise, }
\end{array}\right. \\
& (i, g, j) \rho= \begin{cases}(i, g(j \psi), j \beta) \quad \text { if } j \in \mathbf{d} \beta, \\
0 \text { otherwise, }\end{cases}
\end{aligned}
$$

whence, by a straightforward argument, we get

$$
\begin{gathered}
\mathbf{d} \delta_{(\lambda, \rho)}=\{(i, g, j) \in S \mid i, j \in \mathbf{d} \beta\} \cup 0 \\
\delta_{(\lambda, \rho)}:(i, g, j) \rightarrow\left(i \beta,(i \psi)^{-1} g(j \psi), j \beta\right) \quad \text { if } i, j \in \mathbf{d} \beta
\end{gathered}
$$

which implies that

$$
\begin{aligned}
{\left[\beta, \iota_{G}, \psi\right] } & =\delta_{(\lambda, \rho)}, \\
\Omega^{\prime}(S) \chi^{-1} & =\{(\xi, \omega, \eta) \in T \mid \omega \in \mathscr{I}(G)\} .
\end{aligned}
$$

9.1. EXAMPLE. Let $Y=\{a, b, c\}$ be a semilattice with $a b=c$. Then with the following notation and multiplication we obtain $\Psi(Y)$ :

$$
\begin{aligned}
& \varepsilon_{a}=\left(\begin{array}{ll}
a & c \\
a & c
\end{array}\right), \quad \varepsilon_{b}=\left(\begin{array}{ll}
b & c \\
b & c
\end{array}\right), \quad \varepsilon_{c}=\left(\begin{array}{l}
c \\
c
\end{array}\right), \\
& \alpha=\left(\begin{array}{ll}
a & c \\
b & c
\end{array}\right), \quad \beta=\left(\begin{array}{ll}
b & c \\
a & c
\end{array}\right), \\
& \varepsilon=\left(\begin{array}{lll}
a & b & c \\
a & b & c
\end{array}\right), \quad \gamma=\left(\begin{array}{lll}
a & b & c \\
b & a & c
\end{array}\right),
\end{aligned}
$$




\begin{tabular}{c|c|c|c|c|c|c|c|} 
& $\varepsilon_{a}$ & $\varepsilon_{b}$ & $\varepsilon_{c}$ & $\alpha$ & $\beta$ & $\gamma$ & $\varepsilon$ \\
\hline$\varepsilon_{a}$ & $\varepsilon_{a}$ & $\varepsilon_{c}$ & $\varepsilon_{c}$ & $\alpha$ & $\varepsilon_{c}$ & $\alpha$ & $\varepsilon_{a}$ \\
\hline$\varepsilon_{b}$ & $\varepsilon_{c}$ & $\varepsilon_{b}$ & $\varepsilon_{c}$ & $\varepsilon_{c}$ & $\beta$ & $\beta$ & $\varepsilon_{b}$ \\
\hline$\varepsilon_{c}$ & $\varepsilon_{c}$ & $\varepsilon_{c}$ & $\varepsilon_{c}$ & $\varepsilon_{c}$ & $\varepsilon_{c}$ & $\varepsilon_{c}$ & $\varepsilon_{c}$ \\
\hline$\alpha$ & $\varepsilon_{c}$ & $\alpha$ & $\varepsilon_{c}$ & $\varepsilon_{c}$ & $\varepsilon_{a}$ & $\varepsilon_{a}$ & $\alpha$ \\
\hline$\beta$ & $\beta$ & $\varepsilon_{c}$ & $\varepsilon_{c}$ & $\varepsilon_{b}$ & $\varepsilon_{c}$ & $\varepsilon_{b}$ & $\beta$ \\
\hline$\gamma$ & $\beta$ & $\alpha$ & $\varepsilon_{c}$ & $\varepsilon_{b}$ & $\varepsilon_{a}$ & $\varepsilon$ & $\gamma$ \\
\hline$\varepsilon$ & $\varepsilon_{a}$ & $\varepsilon_{b}$ & $\varepsilon_{c}$ & $\alpha$ & $\beta$ & $\gamma$ & $\varepsilon$ \\
\hline
\end{tabular}

$$
\begin{aligned}
& \Theta(Y)=\left\{\varepsilon_{a}, \varepsilon_{b}, \varepsilon_{c}\right\}, \\
& \Phi(Y)=\Theta(Y) \cup\{\alpha, \beta\}, \\
& \Omega^{\prime}(Y)=\Theta(Y) \cup\{\varepsilon\}, \\
& \Psi(Y)=\Theta(Y) \cup\{\alpha, \beta, \gamma, \varepsilon\} .
\end{aligned}
$$

\section{REFERENCES} 56-64.

1. J. E. Ault, The translational hull of an inverse semigroup, Glasgow Math. J. 14 (1973),

2. A. H. Clifford, Semigroups admitting relative inverses, Ann. of Math. 42 (1941), 10371049.

3. A. H. Clifford and G. B. Preston, The algebraic theory of semigroups, Vol. I, Math. Surveys No. 7, Amer. Math. Soc. (Providence, R.I., 1961).

4. W. D. Munn, Fundamental inverse semigroups, Quart. J. Math. Oxford Ser. 221 (1970), 157-170.

5. M. Petrich, Introduction to semigroups (Merrill, Columbus, 1973).

6. M. Petrich, Extensions normales de demi-groupes inverses, Fund. Math. (to appear).

7. I. S. Ponizovskǐ̌, A remark on inverse semigroups, Uspehi Mat. Nauk. 20 (1965), 147-148 (Russian).

8. N. R. Reilly, The translational hull of an inverse semigroup, Canad. J. Math. 26 (1974), 1050-1068.

9. V. M. Širjajev, On a class of inverse semigroups, Vestnik Beloruss. Gos. Univ. Ser. I 2 (1970), 10-13 (Russian).

UNIVERISTÉ DE MONTPELLIER

FrANCE 